\title{
Effects of inducible overexpression of DNp73 $\alpha$ on cancer cell growth and response to treatment in vitro and in vivo
}

\author{
M Marabese ${ }^{*, 1}$, S Marchini ${ }^{1}$, MA Sabatino ${ }^{1}$, F Polato ${ }^{1}$, \\ F Vikhanskaya ${ }^{1}$, E Marrazzo $^{1}$, E Riccardi ${ }^{2}$, E Scanziani $^{2}$ and \\ M Broggini ${ }^{1}$ \\ 1 Laboratory of Molecular Pharmacology, Istituto di Ricerche Farmacologiche \\ 'Mario Negri', Via Eritrea 62, 20157 Milan, Italy \\ ${ }^{2}$ Dipartimento di Patologia Animale, Università degli Studi di Milano, Milan, Italy \\ * Corresponding author: M Marabese, Laboratory of Molecular Pharmacology, \\ Istituto di Ricerche Farmacologiche 'Mario Negri', Via Eritrea 62, 20157 Milan, \\ Italy. Fax: + 39-02-3546277; E-mail: marabese@ marionegri.it
}

Received 12.7.04; revised 28.12.04; accepted 26.1.05; published online 06.5.05 Edited by G Melino

\begin{abstract}
The p73 gene has a complex regulation, which leads to the expression of different isoforms, often with opposite biological effects. We have generated in the human colocarcinoma cell line HCT116, expressing a wild-type p53, an inducible DNp73 $\alpha$ expressing system. Two clones (HCT116/DN3 and HCT116/DN14), upon doxycycline addition, show a strong expression of DNp73 $\alpha$. In vitro the two DNp73 $\alpha$ overexpressing clones grow at similar rate of the control transfected clone (HCT116/8a) and similarly respond to DNA damage. When injected in mice, HCT116/DN3, HCT116/DN14, and HCT116/8a cells grew similarly in the absence or presence of tetracycline. In HCT116/DN3 and HCT116/DN14 tumors, tetracycline induced a strong expression of DNp73 $\alpha$ both as mRNA and protein. These results indicate that in this system the overexpression of the DNp73 $\alpha$ does not induce a more aggressive phenotype and does not seem to be associated with a reduced response of the cells to treatment with anticancer agents.
\end{abstract}

Cell Death and Differentiation (2005) 12, 805-814.

doi:10.1038/sj.cdd.4401622

Published online 6 May 2005

Keywords: p73; anticancer agents; in vivo; p53

Abbreviations: cDDP, cis-dichloro-diammine platinum; IHC, immunohistochemistry; doxy, doxycycline

\section{Introduction}

p73 has been identified as a structural and functional homologue of the tumor suppressor protein p53. ${ }^{1}$ The two proteins share similarities in the DNA binding domain and both can act as transcription factors. ${ }^{2}$ Despite this similarity, the two proteins are likely to have distinct functions, particularly in tumor formation and progression. ${ }^{3-5}$ From a structural point of view, p73 is characterised by the presence of different isoforms derived from alternative splicing (termed $\alpha$ to $\zeta$ ), which share the same amino-terminal and central DNA binding domain, but differ in a variety of carboxy terminal portions. ${ }^{1,6,7}$ In addition to the C-terminal variants, different $\mathrm{N}$-terminal truncated isoforms (DNp73, DN'p73, DEx3p73 and DEx2/3p73) have been identified further increasing the complexity of the p73 family. ${ }^{8-11}$ All these isoforms lack the transactivation domain. Interestingly, one of the $\mathrm{N}$-truncated isoforms, the $\mathrm{DNp} 73$, arises from the use of an alternative promoter present in intron 3 , which contains a p53 responsive element in its DNA sequence and which has been reported to functionally respond to both p53 and TAp73. ${ }^{12,13}$ The Nterminal truncated isoforms (including $\mathrm{DNp} 73 \alpha$ ) have been reported to act as dominant-negative regulators on TAp73 isoforms (and on p53). ${ }^{11,14,15}$ Acting as a dominant-negative inhibitor, DNp73 can interfere with the p53 binding to its downstream p53-responsive elements and by inhibiting the transcription of the TAp73 isoforms. This effect is thought to be mediated either by competition through its DNA binding domain, as for p53-responsive elements, and/or by proteinprotein interaction through its oligomerisation domain. Conversely, downregulation of endogenous $\mathrm{DNp} 73 \alpha$ levels by antisense methods enhances p53 and TAp73-mediated apoptosis in tumor cells.

Furthermore, recent data show that upon strong DNA damage DNp73 is rapidly degraded, releasing the block exerted on p53 and TA-p73 and thus allowing cell cycle arrest and apoptosis to proceed. ${ }^{16}$ All these data show that DNp73 is part of a dominant-negative feedback loop that regulates the function of both p53 and TAp73 and that this regulation can be overcome in case of strong DNA damage.

The relative distribution of DNp73 in tumors is therefore likely to be an important determinant of cellular response to treatment. In human neuroblastoma and in human ovarian carcinomas, a correlation between DNp73 expression and survival has been reported. ${ }^{17,18}$ In ovarian cancer, the levels of TAp73 were anyway always higher than those of the $\mathrm{N}$-terminal truncated forms ${ }^{18}$ This suggests that DNp73 (and perhaps other isoforms) could have other functions, distinct from a simple antagonism on the TA isoform.

On the basis of these considerations, we decided to generate clones overexpressing $\mathrm{DNp} 73 \alpha$ protein in the colocarcinoma cell line HCT-116. With these clones we have analysed the effects of inducible ectopic expression of DNp73 on cell behaviour in vitro and in vivo, with the aim of better understanding the role of this specific isoform in determining cellular response to treatment.

\section{Results}

Transfection of HCT116 cells with the DNp73 $\alpha$ plasmid subcloned in the tetracycline inducible system, resulted in 
different clones growing in antibiotic selection media. Two of these, namely clones HCT116/DN3 and HCT116/DN14 were selected for further characterisation. These two clones showed, with real time RT-PCR, a strong tetracycline dependence with a 15- to 30-fold increase in the DNp73 $\alpha$ mRNA levels upon addition of tetracycline or doxycycline (doxy) in the medium if compared with the not induced clones (Figure 1a). An even higher increase was found when the RNA expression was compared with the control clone HCT116/8a. This strong induction was also observable at protein level as shown in Figure $1 \mathrm{~b}$, where in the presence of doxy the $\mathrm{DNp} 73 \alpha$ protein was nicely detectable in both HCT116/DN3 and HCT116/DN14 clones but not in the control clone HCT116/8a. In all clones, in the absence of doxy, the levels of $\mathrm{DNp} 73 \alpha$ were almost undetectable. Furthermore, immunofluorescence staining of cells in the absence or in the presence of doxy did show that the HCT116/DN3 and HCT116/DN14 clones express DNp73 $\alpha$ in the nuclei (Figure 1c). The induction of the expression was very rapid, being measurable as early as $3 \mathrm{~h}$ upon addition of doxy and was maintained for at least $24 \mathrm{~h}$ following removal of doxy from the medium (Figure 1d). The induction was, at least for one clone (HCT116/DN14), dependent on the concentration of doxy, as shown in Figure 1e, where it can be seen that between 0.1 and $1 \mathrm{ng}$ of doxy per $\mathrm{ml}$ of medium a progressive increase in the levels of DNp73 $\alpha$ is achievable.

To verify whether the increased intracellular levels of DNp73 $\alpha$ were also functionally active, we checked its ability to inhibit p53 function. PG13Luc and p21Luc plasmids, containing different p53 responsive elements $5^{\prime}$ to the luciferase gene, were transiently transfected into the HCT116/8a, HCT116/DN3 and HCT116/DN14 clones and cells were treated or not with $2 \mu \mathrm{g} / \mathrm{ml}$ of doxy for $24 \mathrm{~h}$. The data reported in Figure $2 \mathrm{a}$ and $\mathrm{b}$ confirmed the ability of induced DNp73 $\alpha$ to compete with p53 in the binding and activation of the transcription of the reported gene. This effect was observable both in a 'pure', artificial p53-responsive promoter (containing only 13 copies of the consensus p53 binding element, PG13luc) (Figure 2a) and in a 'natural' p53responsive promoter isolated from $\mathrm{p} 21$ genomic sequences (Figure 2b). To further address this point, we determined the endogenous mRNA levels of two p53 downstream genes, p21 (Figure 2c) and bax (Figure 2d) in untreated or UV-damaged cells, either in the absence or presence of doxy, in the different clones. In untreated cells, the addition of doxy slightly increases the endogenous p21 and bax levels (possibly

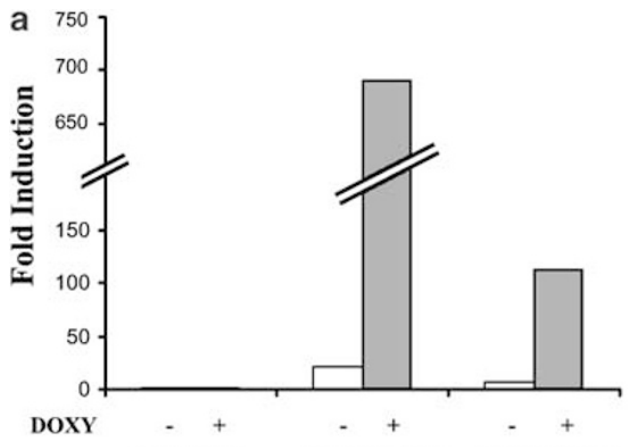

b
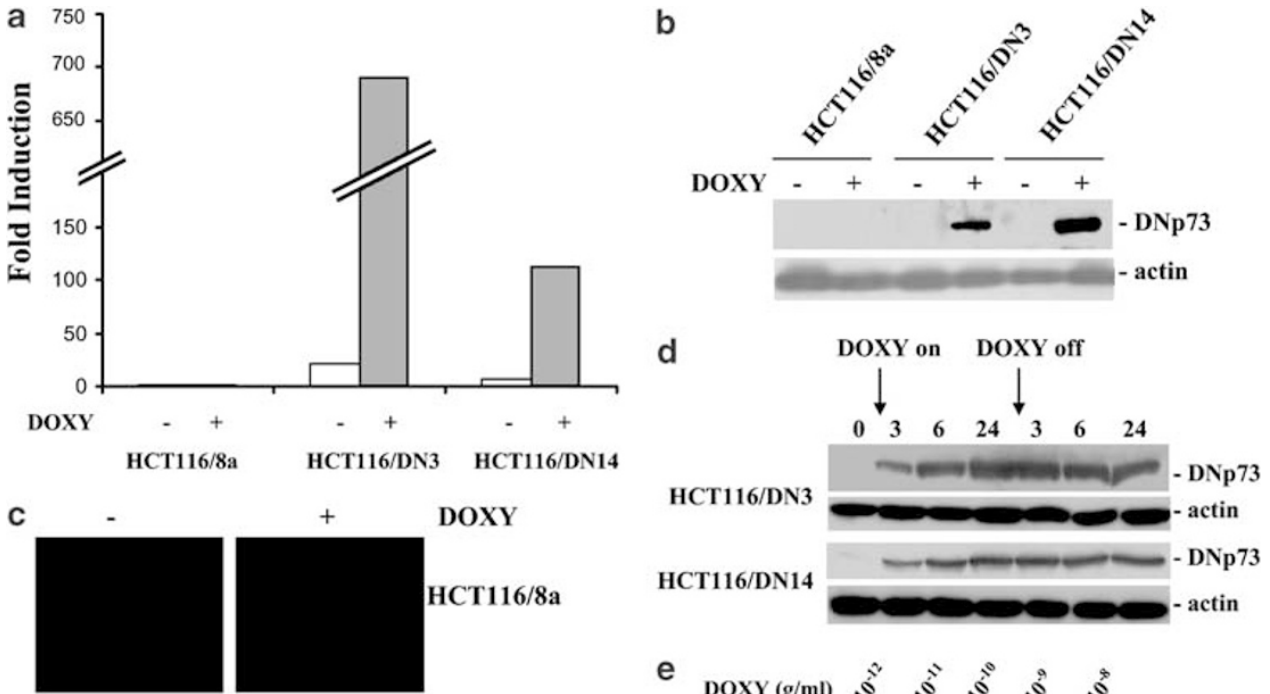

DOXY
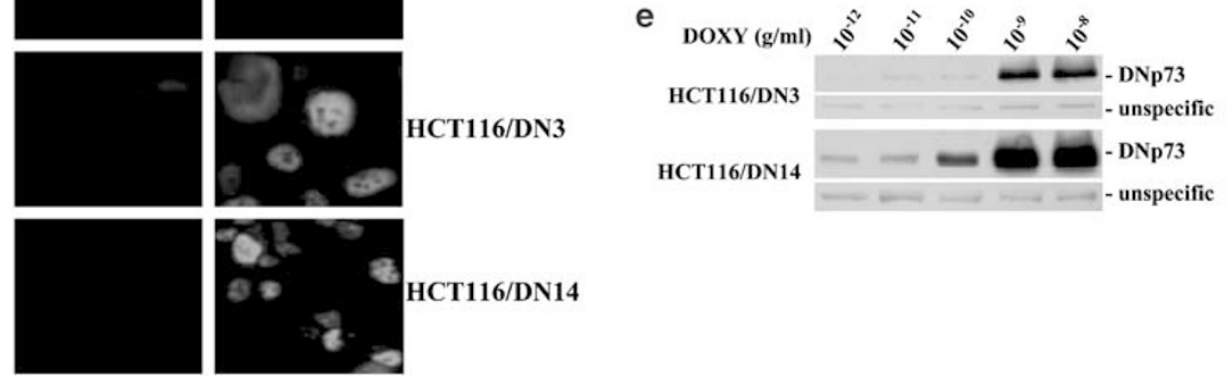

Figure 1 Characterisation of DNp73 $\alpha$ expressing clones. (a) Relative mRNA expression of DNp73 $\alpha$ in HCT116/8a, HCT116/DN3 and HCT116/DN14 clones following doxy treatment $(2 \mu \mathrm{g} / \mathrm{ml})$. mRNA levels were determined by real-time RT-PCR $24 \mathrm{~h}$ following the addition of doxy. Expression of clone HCT116/8a in the absence of doxy was arbitrarily set to 1. (b) Western blot analysis of expression of DNp73 $\alpha$ in HCT116/8a, HCT116/DN3 and HCT116/DN14 cells in the absence or in the presence of doxy $(2 \mu \mathrm{g} / \mathrm{ml})$ for $24 \mathrm{~h}$. Actin was used as internal control. (c) Immunofluorescence staining of HCT116/8a, HCT116/DN3 and HCT116/DN14 cells untreated or treated for $24 \mathrm{~h}$ with doxy $(2 \mu \mathrm{g} / \mathrm{ml})$. (d) Kinetic of the induction of DNp73 $\alpha$ in HCT116/DN3 and HCT116/DN14 clones following the addition of $2 \mu \mathrm{g} / \mathrm{ml}$ of doxy. Total proteins were extracted at the indicated time points following the addition and removal of doxy. Actin was used as internal control. (e) Effect of doxy concentration on the expression of DNp73 $\alpha$ in HCT116/DN3 and HCT116/DN14 cells. Total proteins were extracted $24 \mathrm{~h}$ after the addition of the indicated amounts of doxy. The presence of an unspecific band at low molecular weight was used as internal control 
a

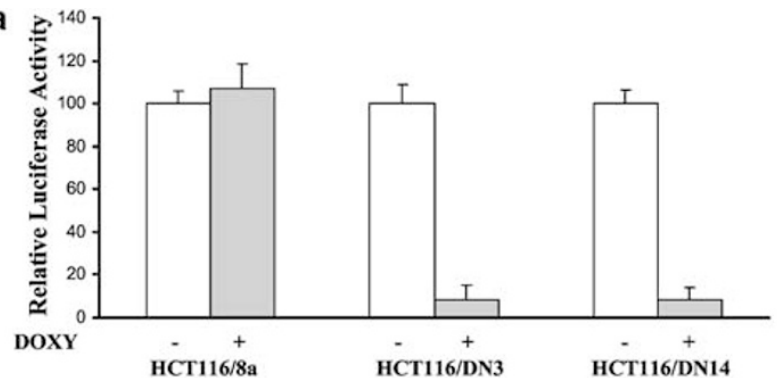

b
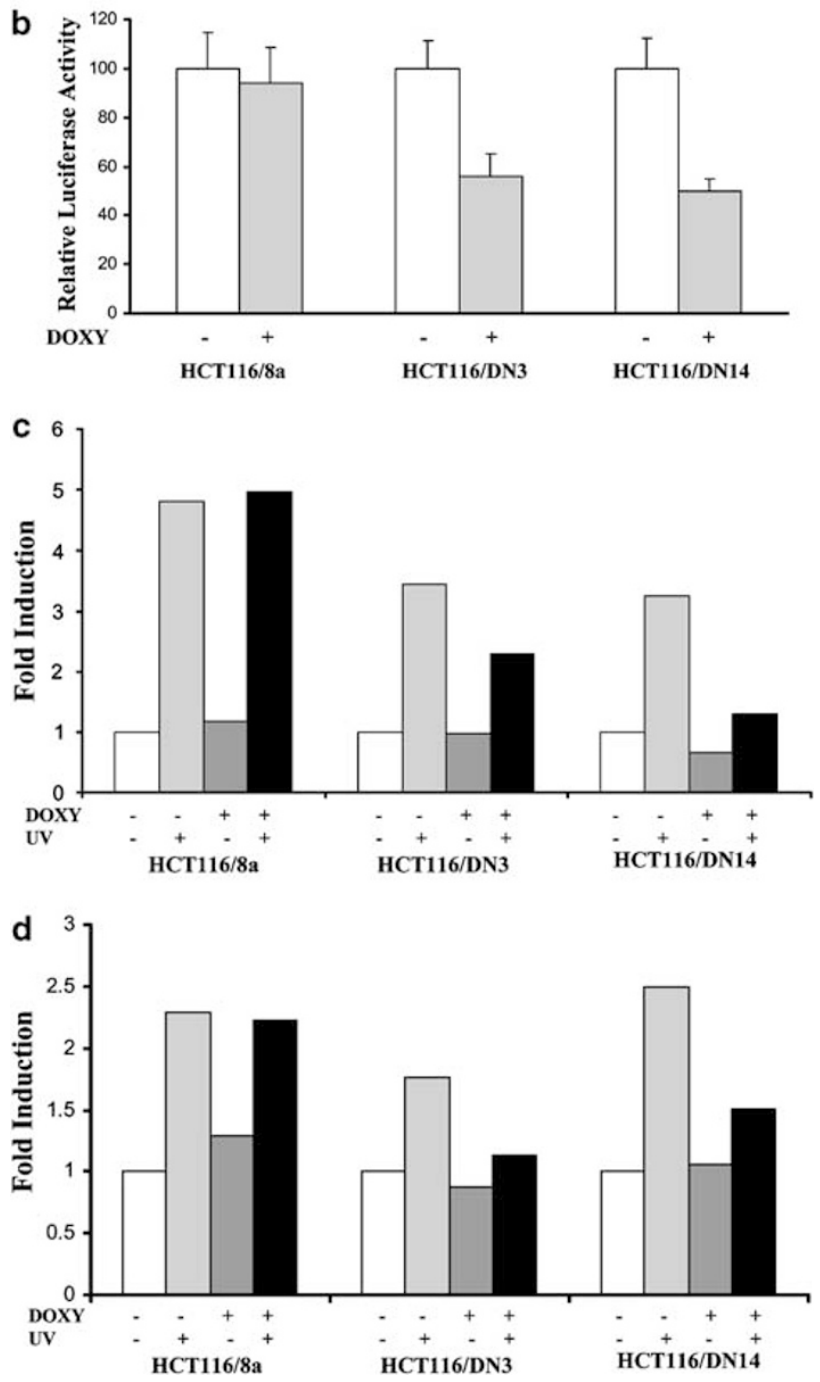

Figure 2 Functional activity of DNp73 $\alpha$ in HCT116/DN3 and HCT116/DN14 cells. Luciferase assay: cells were transfected with pG13Luc (a) and p21Luc (b) construct and luciferase activity determined $24 \mathrm{~h}$ after in the absence or in the presence of $2 \mu \mathrm{g} / \mathrm{ml}$ of doxy. Values are expressed as percentage of luciferase activity normalised with renilla activity. Luciferase activity for each clone determined in the absence of doxy was set to $100 \%$. Each graph represents the mean \pm S.D. of at least three determinations. Real-time RT-PCR determination: endogenous p21 (c) and bax (d) mRNA levels were determined to verify the ability of induced DNp73 $\alpha$ to interfere with regulation of two p53 responsive genes. In each graph basal levels of $p 53$ responsive genes were arbitrary set to 1. Endogenous p21 and bax mRNA levels in HCT116/8a, HCT116/DN3 and HCT116/DN14 cells untreated (white bars) or treated with 20J of UV light (light grey bars) were measured $24 \mathrm{~h}$ after irradiation. The induction of DNp73 $\alpha$ in untreated (dark grey bars) or treated cells with 20J of UV light (black bars) was carried out by addition of doxy $2 \mu \mathrm{g} / \mathrm{ml} 24 \mathrm{~h}$ before treatment through the activation of p53) in HCT116/8a cells, while in the two DNp73 $\alpha$ clones no change or a slight decrease in mRNA levels upon doxy induction was observed. When p21 and bax levels were measured in the clones following damage, we found that UV treatment was able to induce (in the absence of doxy) p21 and bax mRNA accumulation both in control clone and in DNp73 $\alpha$ clones. The addition of doxy $24 \mathrm{~h}$ before DNA damage decreased the ability of p53 to fully induce the expression of these genes in both $\mathrm{DNp} 73 \alpha$ clones, being ineffective in the mock transfected clone.

Having found that the two DNp73 $\alpha$ clones do express a functional $\mathrm{DNp} 73 \alpha$ following induction, we tested the response to DNA damage induction in vitro. We first analysed the growth of these clones in the absence and presence of doxy, finding that the addition of doxy does not modify the growth of both mock transfected and DNp73 $\alpha$ expressing clones (Figure 3a). The HCT116/DN14 clone seems to grow slightly faster than the other DNp73 $\alpha$ expressing clone and control cells, but the analysis of doubling times showed that there were not significant differences in the growth rate even though HCT116/DN3 was a bit slower than other clones (Figure 3b). These experiments were performed by seeding the cells and adding doxy immediately after. The expression of DNp73 $\alpha$ in HCT116/8a, HCT116/DN3 and HCT116/DN14 clones following doxy induction was checked by Western blotting at all time points used for determining cell growth and the DNp73 $\alpha$ protein was nicely detectable till the end of the experiment (Figure 3c). Furthermore, cell cycle phase distribution in these cells before and after induction with doxy did not reveal any significant difference (see supplementary material, annex 1 panels $\mathrm{A}-\mathrm{C}$ ).

We then tested the ability of these cells to respond to DNA damage induction in the presence or in the absence of doxy. Three different kinds of damage, that is, exposure to UV light, treatment with the alkylating agent cis-dichloro-diammine platinum (CDDP) or with the intercalating agent doxorubicin were selected. All these treatments were given when the DN forms were already expressed and the results obtained shown in Figure 4. As it can be seen, a concentration dependent inhibition of the growth was found for UV, cDDP or doxorubicin. When the treatments were performed in the presence of doxy, no difference were obtained, indicating that, at least in these cells, the presence of high levels of DNp73 $\alpha$ does not result in a modification of cellular response to damage.

It is to note that in another different cancer cell line (A2780, derived from a human ovarian carcinoma) we selected two clones stably overexpressing $\mathrm{DNp} 73 \alpha$, and again we found that these clones do respond to treatment with different anticancer agents similarly to parent cells (data not shown).

Differently from what reported in other cellular systems, such as Saos-2, in our clones DNp73 $\alpha$ was not rapidly degraded after DNA damage. In Figure 5a, in fact, it can be seen that the induced protein remains almost unchanged in cells till $24 \mathrm{~h}$ after the treatment.

To verify that the p53 pathway was intact in the clones, we transfected in these cells a wild-type p53 expressing plasmid. As shown in Figure $5 \mathrm{~b}$, wild-type 553 overexpression in all the clones induced a strong growth inhibition.

To check whether the presence of high levels of DNp73 $\alpha$ could alter the growth of these cancer cells in vivo, we injected 

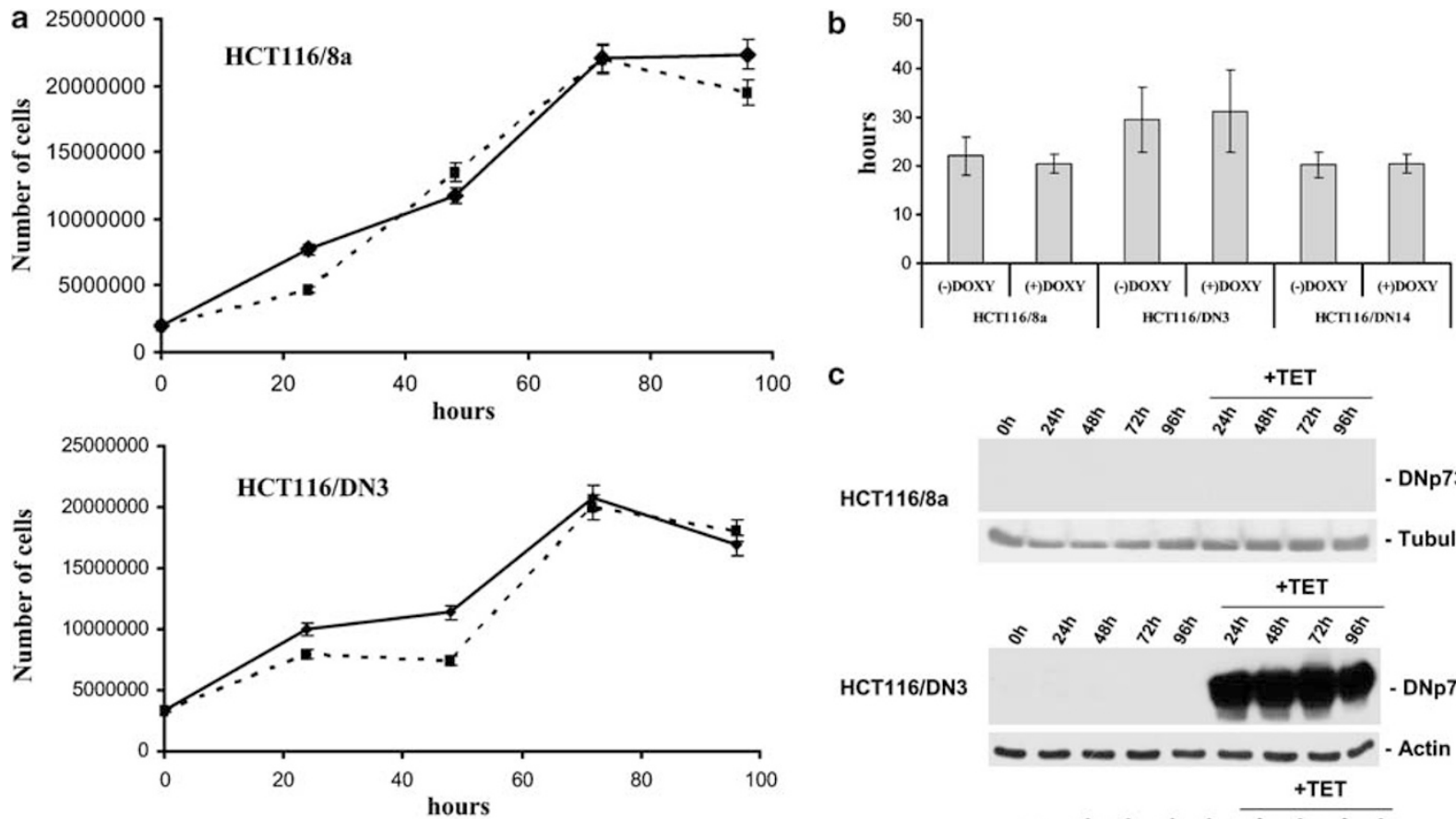

C

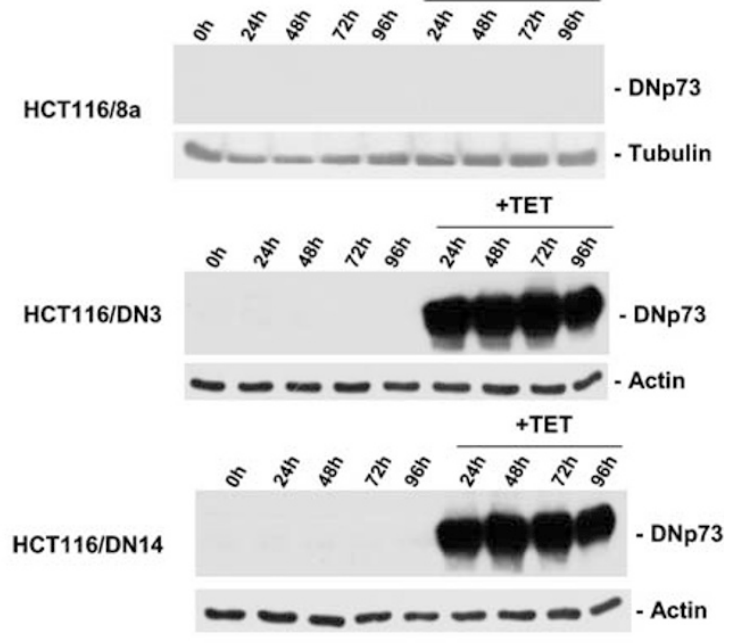

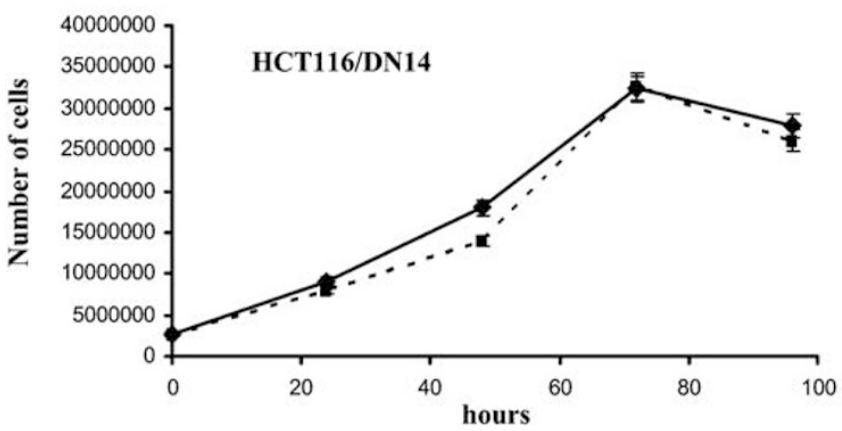

Figure 3 In vitro growth of clones. (a) In vitro growth of HCT116/8a, HCT116/DN3 and HCT116/DN14 cells in the absence (continuous line) or in the presence of $2 \mu \mathrm{g} / \mathrm{ml}$ of doxy (hatched line). Cells were seeded in $10 \mathrm{~cm}$ plates and counted every $24 \mathrm{~h}$ following the addition of doxy. Values represent the mean of four samples \pm S.D. (b) Plot represents the doubling times in hours for each clone in the absence or in the presence of doxy. (c) Western blot analysis of expression of DNp73 $\alpha$ in HCT116/8a, HCT116/DN3 and HCT116/DN14 cells, seeded in the same conditions and time points used to determine the growth curve reported in (a), in the absence or in the presence of doxy $(2 \mu \mathrm{g} / \mathrm{ml})$. Actin was used as internal control

HCT116/8a, HCT116/DN14 and HCT116/DN3 clones in nude mice. Half of the animals were subjected to treatment with tetracycline by adding the antibiotic in the drinking water, replacing the solution every other day, starting when the tumor was palpable and the treatment continued until the mice were killed.

The tumor takes for all clones either in the presence of in the absence of tetracycline was $100 \%$. Figure $6 a$ reports the results obtained in a typical experiment. When the growth of the tumors was compared in animals receiving or not tetracycline, we did not find any difference in the growth rate in all the three clones. A slightly (although not statistically significant) increase in the growth rate for HCT116/DN14 was observed in the group overexpressing the DNp73 $\alpha$ isoform.

HCT116/DN14 tumors reached a slightly higher tumor weight than parental cells (either in the absence or in the presence of tetracycline). The other DNp73 $\alpha$ overexpressing clone (HCT116/DN3) had instead a slightly slower growth. The doubling times calculated from all the growth curves, and reported in Figure $6 b$, are however rather similar.

Figure $6 \mathrm{c}$ shows that the mice bearing the HCT116/DN14 or the HCT116/DN3 clone treated with tetracycline do express high levels of DNp73 $\alpha$, while these levels were almost undetectable in the absence of tetracycline. In the parental HCT116/8a clone the levels of DNp73 $\alpha$ were undetectable either in the presence or in the absence of tetracycline. The expression of DNp73 $\alpha$ was not only detectable at the end of the experiment but also 14 days following tumor implant, when tumor biopsies were randomly taken from mice bearing the different tumors and untreated or treated with tetracycline. The localisation of the DNp73 $\alpha$ expressed in HCT116/DN14 or HCT16/DN3 tumor bearing mice was determined by immunohistochemistry $(\mathrm{IHC})$ in sliced obtained from the same tumors used for determining the growth and the DNp73 $\alpha$ expression by Western blotting. The results obtained showed that the 
HCT116/8a
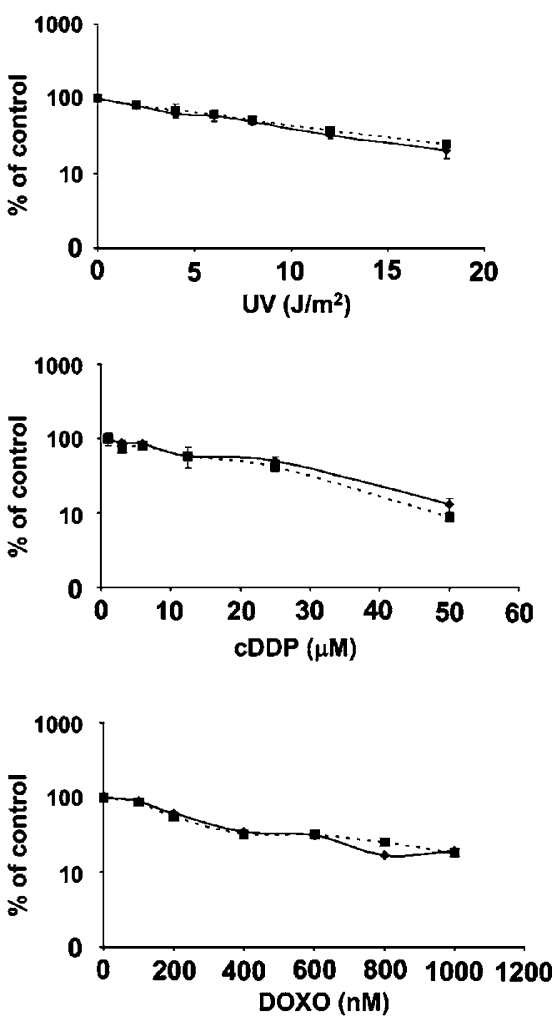

HCT116/DN3

UV LIGHT
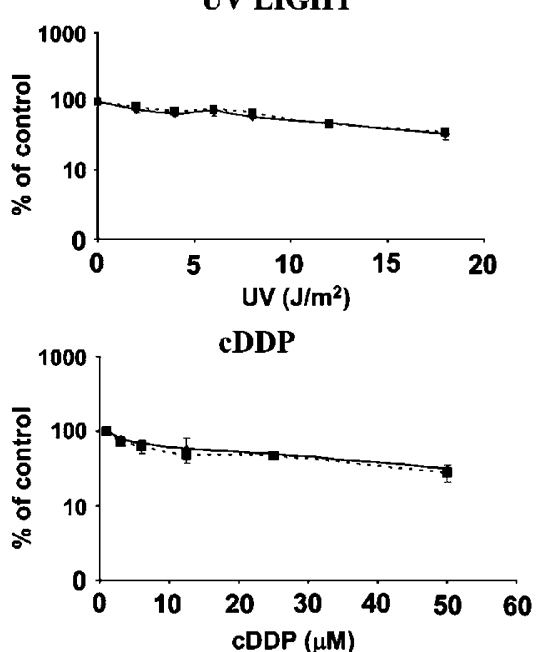

DOXORUBICIN

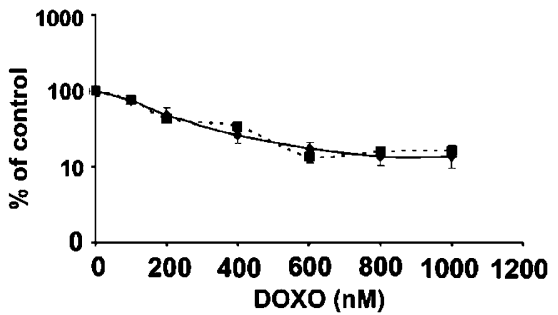

HCT116/DN14
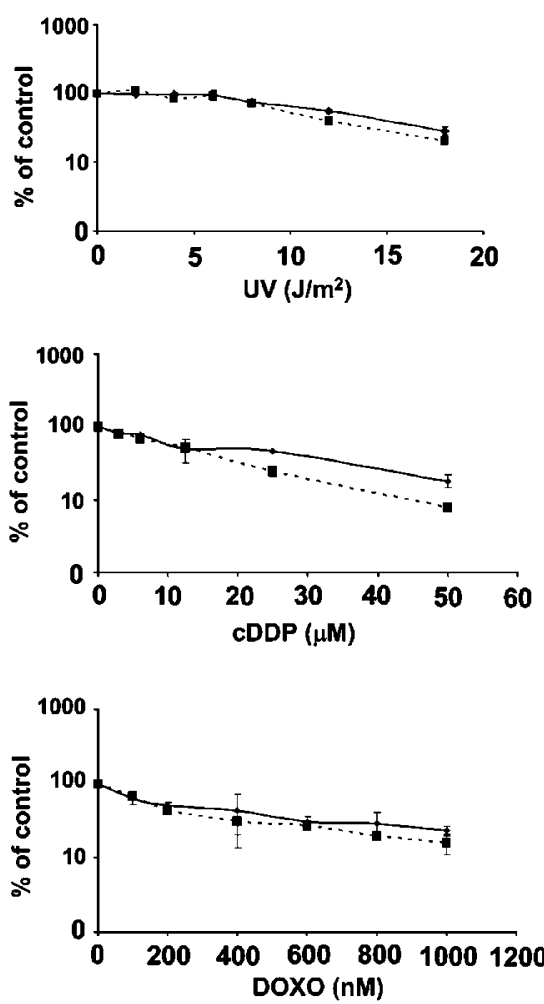

Figure 4 In vitro cytotoxicity. Response of HCT116/8a, HCT116/DN3 and HCT116/DN14 cells to treatment with UV (upper panel), CDDP (middle panel) or doxorubicin (lower panel). Cells were seeded in six-well plates and grown one day in free drugs and doxy medium. The second day after pleating, cells were incubated o.n. in the absence (continuous line) or in the presence of $2 \mu \mathrm{g} / \mathrm{ml}$ of doxy (hatched line). Treatments were performed after doxy incubation at the indicated drug concentrations or UV doses. The number of colonies formed were stained with $10 \%$ crystal violet in $20 \%$ ethanol and automatically counted on an image analyser. The data of the survival curves were plotted as percentages of untreated controls. Each experiment consisted of at least three replicates \pm S.D. The number of colonies counted in untreated samples were $262 \pm 49,311 \pm 53$ and $308 \pm 25$ for HCT116/8a, HCT116/DN3 and HCT116/DN14 cells, respectively

expression of DNp73 $\alpha$ is well detectable in the nuclei (data not shown).

When the activity in vivo of cDDP was evaluated in the different tumors (Figure 6d), we found that the drug was able to induce a $40-50 \%$ inhibition of the growth at the doses used. The activity of cDDP was similar in the three different clones and independent from the absence of presence of tetracycline.

To test whether the absence of hMLH1 expression in HCT116 could have any influence in the results, we transiently transfected hMLH1 cDNA in these cells (Figure 7). Expression of hMLH1 in these clones, well detectable by Western blotting (panel b) is associated, as expected, to a slightly higher activity of cDDP. However, this increase was observed in all the three clones, either in the absence or in the presence of tetracycline, suggesting that this effect is independent from the expression of DNp73 $\alpha$ (Figure 7a, c).

\section{Discussion}

There are now many evidences supporting that p53 and p73 do have different roles in tumorigenesis. The lack of mutations in p73 gene in human tumors and the finding that p73 knockout mice do not develop tumors are strong evidences against a role of p73 as a tumor suppressor. ${ }^{3,19-23}$ Recent evidences showed that p73 interacts with mutant p53 and this interaction can be an important determinant of chemosensitivity. ${ }^{24,25}$

The p73 gene has a complex genomic structure, which account for the expression of different isoforms. Increasing evidences suggest that the isoforms lacking the $\mathrm{N}$-terminus of p73 (i.e. the transactivation domain) act as dominant-negative regulators of both TAp73 and p53. ${ }^{11,14,26}$

In human tumors, it has been reported that not only p73 is not mutated but rather is frequently overexpressed compared to normal adjacent tissues. The majority of the results, however, were obtained using methods not able to discriminate between the different isoforms with opposite effects and therefore do not help in clarifying the role of TAp73 versus DNp73 in tumorigenesis. Recent papers, however, tried to specifically address this point. In these manuscripts, the level of DN isoforms was found to be higher in those patients with a relatively poorer prognosis. ${ }^{17,18,27}$ In these measurements, however, the levels of TAp73 measured in tumors were still in excess over the DNp73 isoforms. This would argue against an inactivation of TAp73 by DN isoform, being the levels of the latter not sufficient (at least theoretically) to sequester the fully 


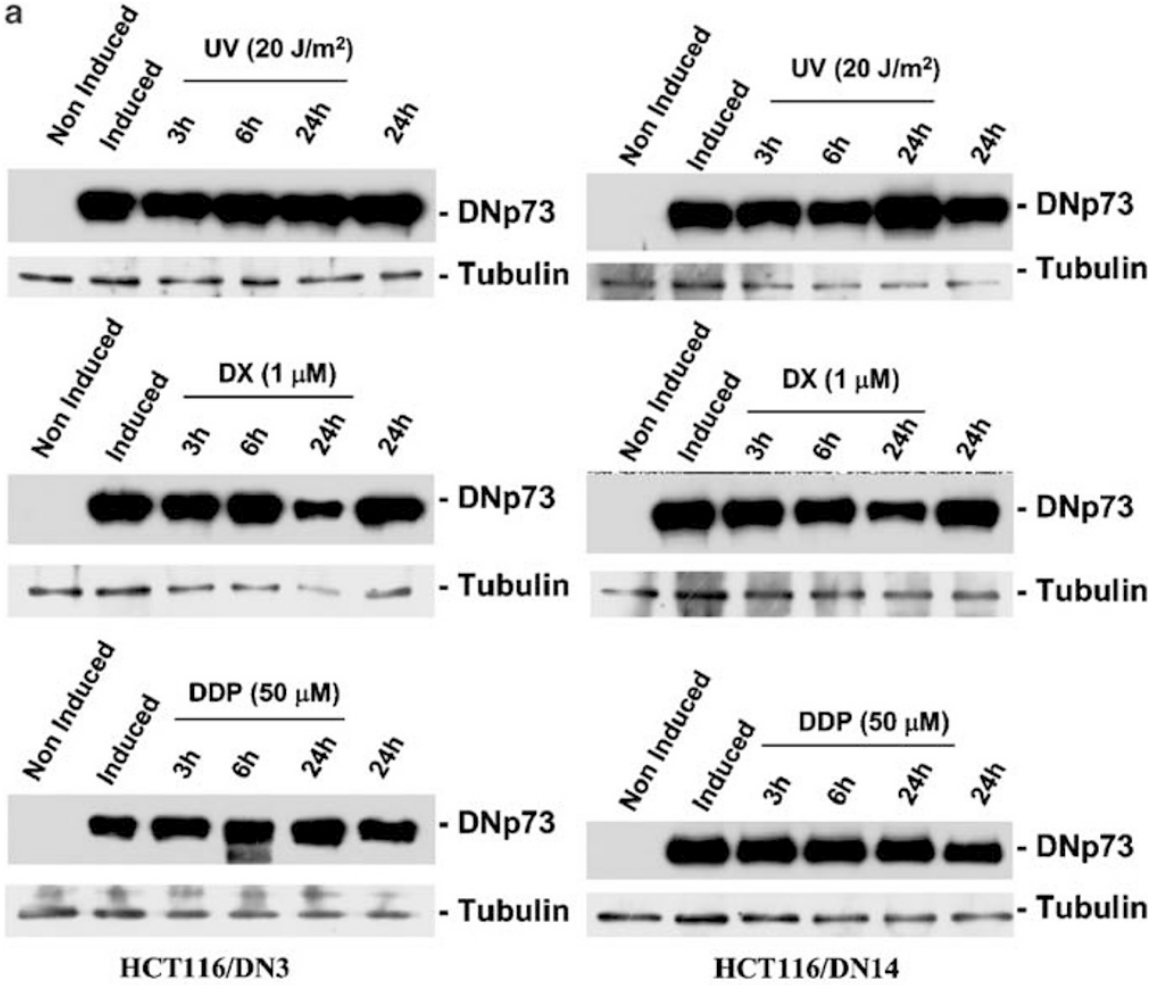

b

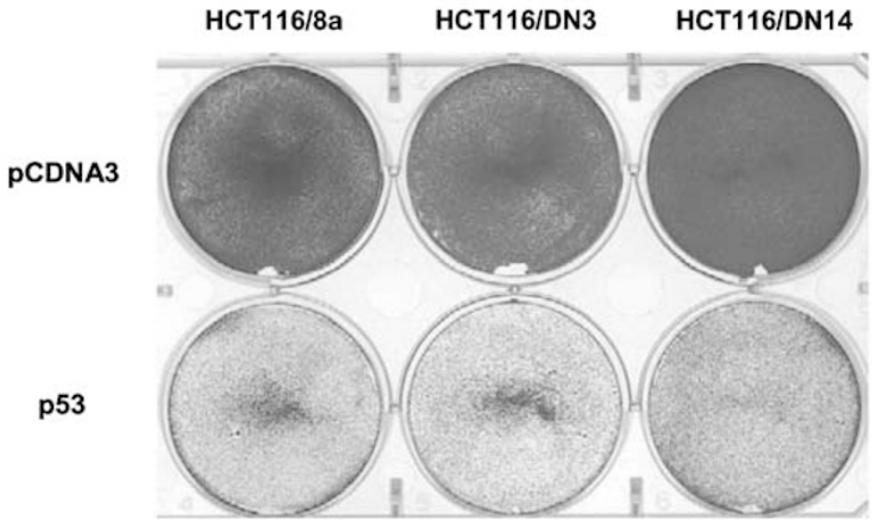

Figure 5 (a) DNp73 $\alpha$ stability after DNA damage treatment. Each clone was induced with doxy $2 \mu \mathrm{g} / \mathrm{ml} \mathrm{o.n.} \mathrm{and} \mathrm{treated} \mathrm{with} \mathrm{the} \mathrm{highest} \mathrm{doses} \mathrm{of} \mathrm{drug} \mathrm{or} \mathrm{UV} \mathrm{light} \mathrm{used}$ for in vitro cytotoxicity experiment. The levels of DNp73 $\alpha$ were assessed before treatment, 3,6 and $24 \mathrm{~h}$ after treatment and at the end of the experiment without treatment. Tubulin was used as internal control. (b) p53 pathway functionality. HCT116/8a, HCT116/DN3 and HCT116/DN14 cells were seeded in six-well plates and transfected with $3 \mu \mathrm{g}$ of pCDNA3 or p53 expressing vector for each well. At 5 days after transfection, plates were stained with $10 \%$ crystal violet in $20 \%$ ethanol

transactive isoforms. It is to note, however, that a stoichio metrically lower level of mutant p53 is sufficient to inactivate the wt 553 , thus suggesting that this could be also the case for the DNp73 isoforms. ${ }^{18}$ Nevertheless, whatever are the relative levels of DN isoforms necessary to act as dominantnegative over the TA isoforms, in our model the levels of DNp73 following induction are well above those of the TA form, which is almost undetectable in this cell line. This, together with the evidences that we have a functional expression of $\mathrm{DNp} 73 \alpha$, as demonstrated by the reduction of p53 ability to transactivate downstream genes, suggests that in these cells $\mathrm{DNp} 73 \alpha$ is indeed blocking the activity of endogenous wtp53 (and TAp73) present in these cells.
The finding that overexpression of $\mathrm{DNp} 73 \alpha$ is not associated to a more malignant phenotype in these cells is surprising, even if these results are not restricted to this system. In another cell line of different origin expressing a wt p53 (A2780 cells, derived from an ovarian cancer), in fact, we produced clones stably overexpressing DNp73 $\alpha$, which show an in vitro growth and a response to damage similar to that of the parent cells (data not shown). Altogether these results are against a clonal selection of cells particularly 'resistant' to the overexpression of $\mathrm{DNp} 73 \alpha$, selection that is per se negligible when inducible clones are used.

A possible explanation for our results is that cancer cells expressing a functional p53 are less susceptible to the 

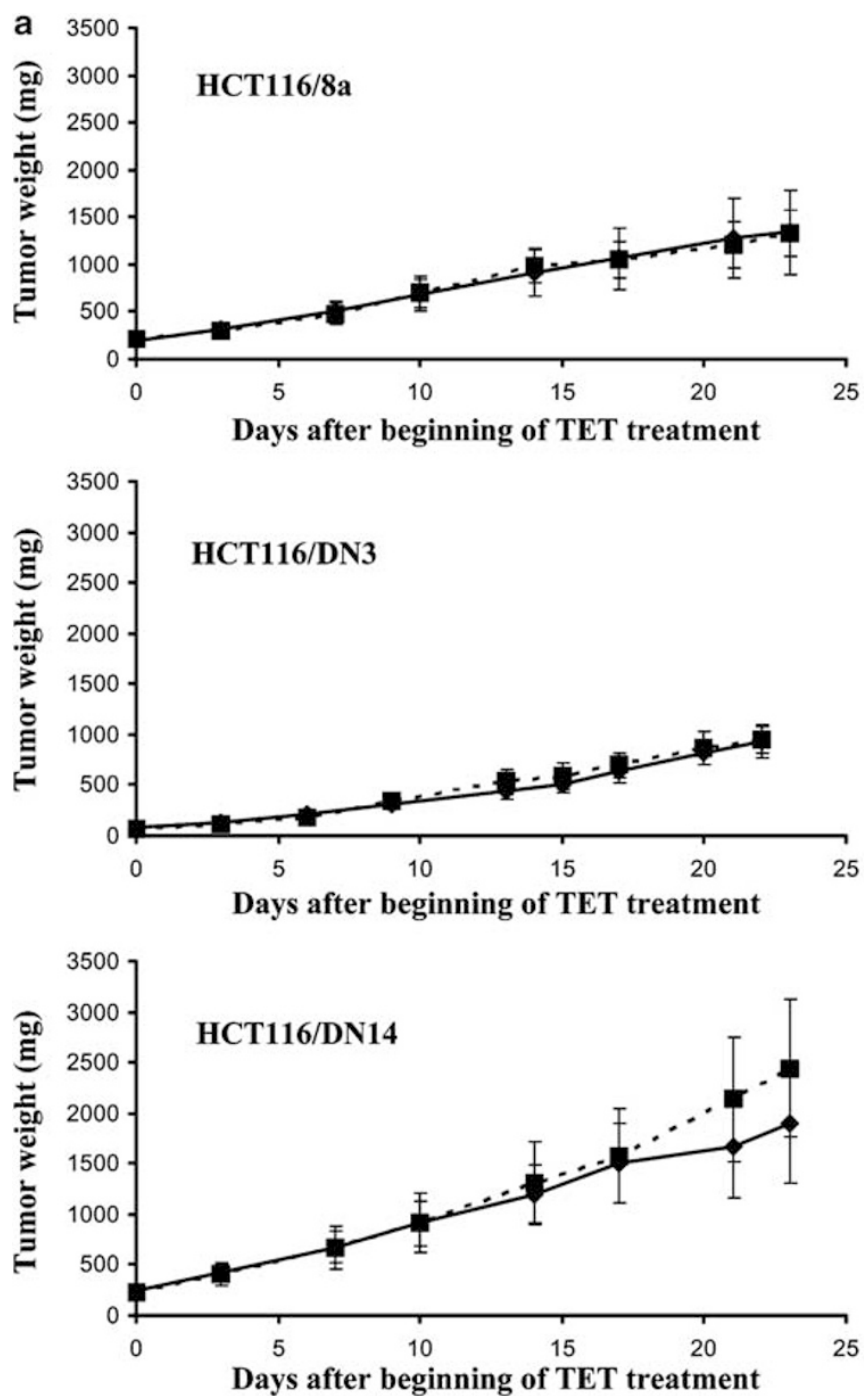

b
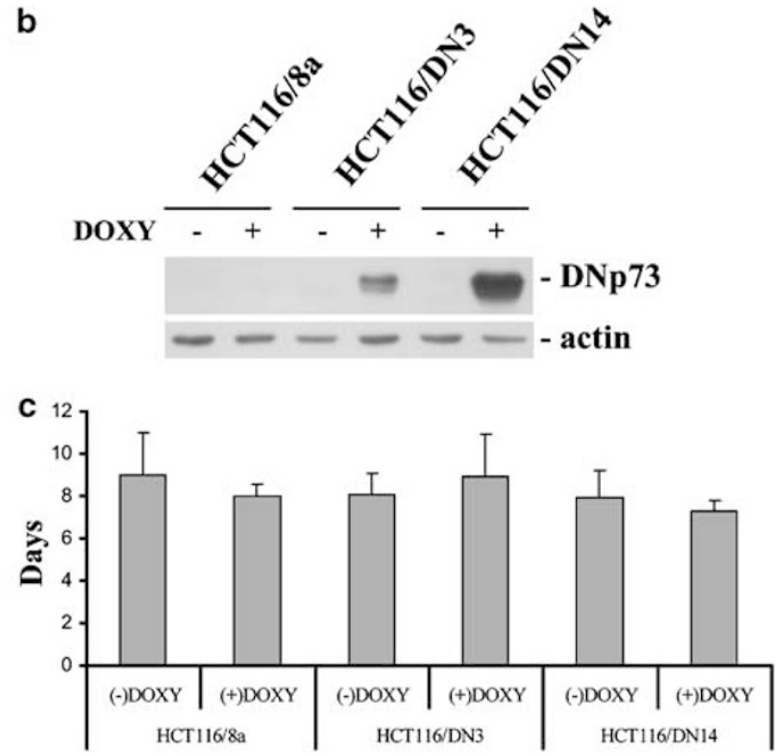

d

$\mathrm{T} / \mathrm{C} \%$

\begin{tabular}{|c|cc|}
\hline \multirow{2}{*}{ HCT116/8a } & -TET & $54 \%$ \\
\cline { 2 - 3 } & +TET & $59 \%$ \\
\hline \multirow{2}{*}{ HCT116/DN3 } & -TET & $65 \%$ \\
\cline { 2 - 3 } & +TET & $58 \%$ \\
\hline \multirow{2}{*}{ HCT116/DN14 } & -TET & $62 \%$ \\
\cline { 2 - 3 } & + TET & $59 \%$ \\
\hline
\end{tabular}

Figure 6 In vivo growth of clones. (a) In vivo growth of HCT116/8a, HCT116/DN3 and HCT116/DN14 cells transplanted in nude mice. Cells were cultured in vitro in the absence of doxy and injected in nude mice untreated or pretreated with tetracycline (added in the drinking water at $2 \mathrm{mg} / \mathrm{ml}$ ). The growth of the tumors was assessed every twice weekly by diameter measurement. (b) DNp73 $\alpha$ levels in tumors of mice receiving or not tetracycline evaluated the same day of cDDP treatment. Actin was used as internal control. (c) Evaluation of doubling times calculated from the growth curves of tumors of mice injected with HCT116/8a, HCT116/DN3 and HCT116/DN14 cells untreated or treated with tetracycline. (d) Activity of cDDP in HCT116/8a, HCT116/DN3 and HCT116/DN14 tumor bearing mice. cDDP was administered intravenously three times every 7 days and the $T / C \%$ reported in the figure have been calculated 7 days following the last treatment

presence of DNp73 respect to cells with no p53 (such as the SaoS-2) or expressing a mutated p53. The finding that the p53 pathway is indeed functional in these clones further supports this possibility.

That $\mathrm{DNp} 73 \alpha$ does not induce a more drug resistant phenotype in vitro or a more aggressive tumor in vivo, is not in contrast with the findings that DNp73 effectively cooperates with ras in inducing a malignant transformation. ${ }^{28}$ In normal cells, the presence of high levels of DN isoforms, hence the inactivation of p53 and TAp73, could be detrimental for the maintenance of genomic integrity. In cancer cells with an already altered genotype, this effect could be less relevant, particularly in cells with a wt p53 expression. These cells, which can grow in the presence of a functional p53, are possibly less susceptible to the inactivation of p53 induced by high $\mathrm{DNp} 73 \alpha$ levels. Alternatively, it must be considered that DNp73 $\alpha$, besides its role as p53 and TAp73 antagonist, can have additional effects independent of p53 (or p73). DNp73 has been in fact shown to be able to induce gene transcription. ${ }^{29,30}$

The fact that overexpression of $\mathrm{DNp} 73 \alpha$ does not induce a more aggressive phenotype and is not associated to resistance to cDDP in vivo (at least in these cells) is not due to a defective localisation of the protein. The protein not only is highly expressed in the tumor growing in vivo but is also well localised to the nucleus. The expression was also maintained in the different passages, since biopsies taken at different time points did reveal constant high DNp73 $\alpha$ expression (data not shown).

Furthermore, the results reported in the present manuscript would suggest that $\mathrm{DNp} 73 \alpha$ overexpression is not sufficient 
812

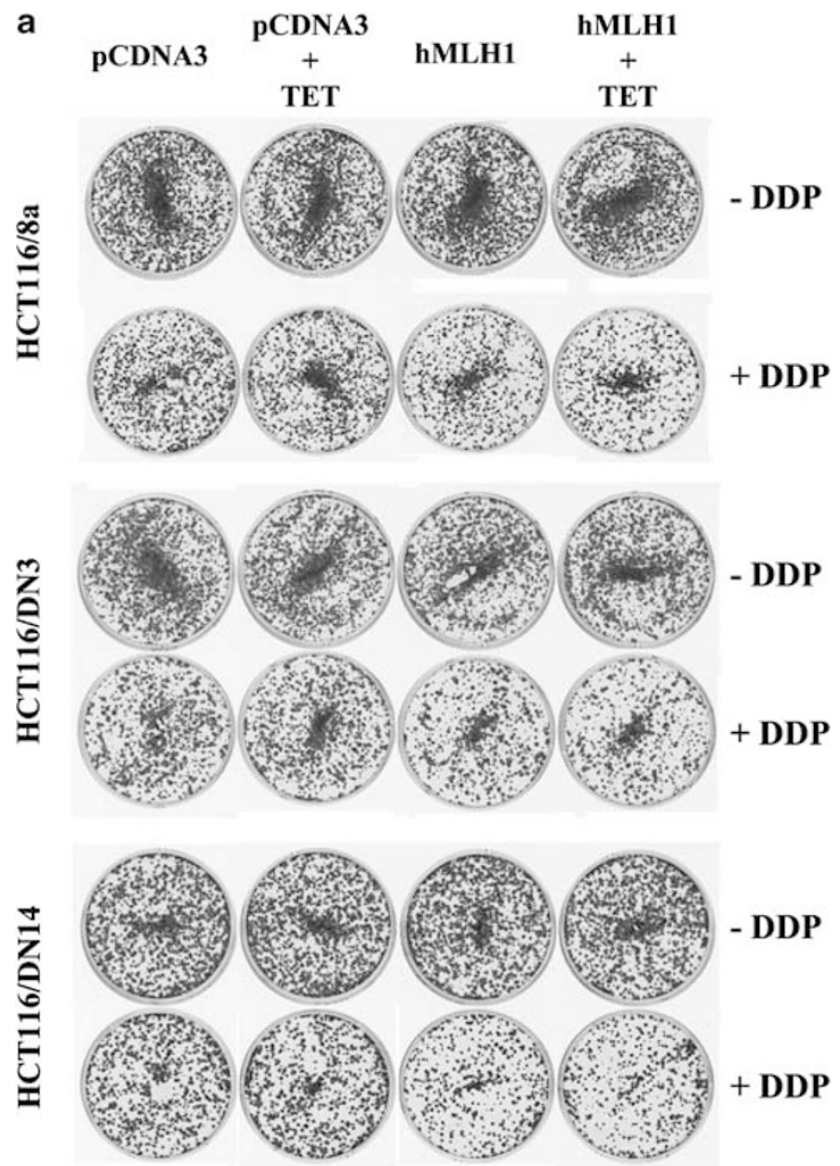

'per se' to induce a resistant or aggressive phenotype. The cell line we selected lacks the expression of hMLH1, reported to be important for the response to DNA damage. ${ }^{5,31}$ However, transient transfection hMLH1 in parental and DNp73 $\alpha$ overexpressing clones slightly enhances the activity of cDDP as expected, but this effect is observable in all the clones, independently from the expression of $\mathrm{DNp} 73 \alpha$. We do not know yet whether the presence of a wt p53 in HCT116 cells could be the reason for these unexpected results. The choice of HCT116 as a model for studying DNp73 $\alpha$ effects was partially driven by the fact that different isogenic subclones derived from parental cells are available,$^{32-34}$ thus offering an almost unique possibility for further studies. Another important point is how the overexpression of this $\mathrm{N}$-truncated isoform changes the gene expression profiles of these cells. This could give us additional information helping in understanding the role and function of this truncated form, particularly being able to use this well-characterised system both in vitro and in vivo.

\section{Materials and Methods}

\section{Cell culture}

The HCT-116 cell line ( $\mathrm{p} 53^{+/+}$), derived from a human colon carcinoma, is routinely maintained in Iscove medium supplemented with $10 \%$ foetal calf serum. A clone transduced with a tetracycline repressor (pcDNA6TR, Invitrogen, CA, USA) was used to generate DNp73 $\alpha$ expressing clones. DNp73 $\alpha$ cDNA (kindly supplied by Dr De Laurenzi) was subcloned in tetracycline inducible plasmid PCDNA4/TO and different clones were picked up. Two of them, clone HCT116/DN3 and clone HCT116/DN14, were selected and allowed to grow in medium supplemented with $10 \%$ of TET System approved foetal bovine serum (BD biosciences, USA) in the presence of $5 \mu \mathrm{g} / \mathrm{ml}$ of blasticidin (Invitrogen), and of $10 \mu \mathrm{g} / \mathrm{ml}$ of zeocin (Invitrogen). As internal control, an HCT116 clone transfected with the empty vector (HCT116/8a) was used. Tetracycline and doxy were purchased from Sigma (Milan, Italy). Transient transfection with wild-type p53 and hMLH1 (kindly supplied by Dr Buermeyer, OHSU Portland, USA) were performed by using Lipofectamine 2000 method (Invitrogen).

C

\begin{tabular}{|l|c|c|c|}
\cline { 2 - 4 } \multicolumn{1}{c|}{} & HCT116/8a & HCT116/DN3 & HCT116/DN14 \\
\hline pCDNA3 & 100.0 & 100.0 & 100.0 \\
\hline pCDNA3+TET & 84.6 & 88.3 & 96.0 \\
\hline hMLH1 & 92.3 & 96.6 & 95.7 \\
\hline hMLH1+TET & 99.8 & 94.1 & 100.3 \\
\hline pCDNA3+DDP & 72.8 & 65.8 & 69.6 \\
\hline pCDNA3+TET+DDP & 64.4 & 62.2 & 65.9 \\
\hline hMLH1+DDP & 58.8 & 57.3 & 56.4 \\
\hline hMLH1+TET+DDP & 57.8 & 49.7 & 55.6 \\
\hline
\end{tabular}

Figure 7 DNA damage clones response in hMLH1 restored clones. (a) HCT116/8a, HCT116/DN3 and HCT116/DN14 cells were seeded in a $10 \mathrm{~cm}$ dish and transfected with pCDNA3 or hMLH1 expressing vector. The day after transfection, cells were detached and plated in $6 \mathrm{~cm}$ dishes. The third day, cells were incubated $0 . \mathrm{n}$. in the absence or in presence of $2 \mu \mathrm{g} / \mathrm{ml}$ of doxy. cDDP treatment was performed after doxy incubation at the concentration of $25 \mu \mathrm{M}$ for $2 \mathrm{~h}$. At 8 days after treatment, colonies were stained with $10 \%$ crystal violet in $20 \%$ ethanol and automatically counted on an image analyser. (b) Western blot analysis of clones transfected with pCDNA3 or hMLH1 expressing vector. Blot shows the hMLH1 levels at the moment of CDDP treatment of previous panel. Lanes HCT116 and HCT116/ch3 were loaded as negative and positive controls, respectively. (c) Quantitative data obtained from the experiment shown in (b) and plotted as percentages of untreated controls

\section{Real time RT-PCR}

In total, $200 \mathrm{ng}$ of total RNA purified with the Trizol protocol (Invitrogen) were retrotranscribed in $20 \mu \mathrm{l}$ with TaqMan Reverse Transcription Kit (Applied Biosystems, UK) and $2 \mu$ were amplified by real-time PCR. Realtime PCR was used for relative quantification of $\mathrm{DNp} 73 \alpha$, while actin was used as internal control.

Primers and TaqMan probe were purchased as ready to use solution (Assay on Demand, Applied Byosystem). Primers and probes sequences to detect the levels of DNp73 $\alpha$ were $5^{\prime}$-GGATTCCAGCATGGACGTCTT$3^{\prime}$ as forward primer and 5'-CGCCTACCATGCTGTACGT-3' and 5'GGCTGCTCATCTGGTCCAT-3' as TaqMan probe (Assay by Design, Applied Biosystem). Reactions were performed in a total volume of $25 \mu \mathrm{l}$ with TaqMan PCR Master Mix following the manufacturer's instructions (Applied Biosystems).

\section{Western blot}

Cell extracts, obtained from untreated and drug treated cells, were prepared by lysing cells in $50 \mathrm{mM}$ Tris- $\mathrm{HCl}$ pH 7.4, $250 \mathrm{mM} \mathrm{NaCl}, 0.1 \%$ 
Nonidet NP-40, $5 \mathrm{mM}$ EDTA, $50 \mathrm{mM} \mathrm{NaF}$ in the presence of aprotinin, leupeptine and phenyl-methyl-sulfonyl-fluoride (PMSF) as proteases inhibitors, for 30 min on ice. Total proteins were purified from snap frozen tumors after homogenation in the same lysis buffer (ratio $1: 1 \mathrm{w} / \mathrm{v}$ ). Insoluble material was pelleted at $13000 \times g$ for $10 \mathrm{~min}$ at $4^{\circ} \mathrm{C}$ and the protein concentration was determined using a Biorad assay kit (BioRad, Italy). In total, $40 \mu \mathrm{g}$ of total cellular proteins were separated on SDSPAGE and electrotransferred to nitrocellulose. Immuno-blotting was carried out with p73 monoclonal antibodies (Oncogene Research, CA, USA) while actin was detected by antiactin polyclonal antibody (Santa Cruz Biotechnology, CA, USA). Antibody binding was revealed by peroxidase labelled secondary antibodies and visualised using enhanced chemioluminescence (Amersham, Italy).

\section{Immunofluorescence}

Cells were plated on glass coverslips and grown for $24 \mathrm{~h}$ in the absence or presence of doxy. After fixing with paraformaldehyde, and permeabilisation with Triton X-100, ${ }^{35}$ cells were stained with $\mathrm{p} 73$ monoclonal antibodies (Oncogene Research) and with fluoresceine-tagged antimouse antibodies (Novocastra). Coverslips were mounted in Mowiol 4-88 (Hoechst, Frankfurt) and observed in an Olympus BX60 fluorescence microscope.

\section{Histological and immunohistochemical analysis}

Tumor samples were fixed in 10\% formalin neutral buffer, routinely processed for histological examination and embedded in paraffin. Haematoxilin and eosin (H\&E) staining was performed for morphological evaluation.

To evaluate $\mathrm{p} 73$ tumoral expression, IHC with $\mathrm{p} 73$ monoclonal antibody (1:200 diluition, Oncogene Research) was performed. Immunoperoxidase staining was performed using an ordinary biotin-streptavidin method. Antigen retrieval was by pressure-cooking in $0.01 \mathrm{M}$ citrate buffer $(\mathrm{pH} 6)$ for $3 \mathrm{~min}$. We used a Vectastain ABC kit (Vector, Burlingame, CA, USA) and AEC (Carbazol) as a chromogen. The sections were then lightly counterstained with haematoxylin. In each immunohistochemical staining we performed additional staining without primary antibody in parallel as negative control.

\section{Luciferase assay}

Cells expressing or not the DNp73 $\alpha$ protein were transfected with $5 \mu \mathrm{g}$ of pG13Luc purified plasmid and $0.5 \mu \mathrm{g}$ of PRL-SV40 (Promega, Italy) as internal normalisation control, using the calcium phosphate technique. ${ }^{36}$ Reporter gene activities were evaluated after $24 \mathrm{~h}$ using the Dual Luciferase System (Promega, Italy). Results are expressed as the percentage of the control luciferase reported activity normalised by the renilla activity value. The mean \pm S.D. of three independent experiments is shown.

\section{In vitro cytotoxicity}

For clonogenic assay, the three different clones were plated at 300 400 cells/well in six-well plates. At $24 \mathrm{~h}$ after seeding, doxy was added to half of the plates and cells were then treated (for $2 \mathrm{~h}$ ) with different concentrations of CDDP or doxorubicin (Sigma) or UV doses. After washing with PBS, plates were incubated for 8-10 days in drug-free medium in the absence or presence of doxy. The number of colonies formed were stained with $10 \%$ crystal violet in $20 \%$ ethanol and automatically counted on an image analyser (Immagini \& Computer, Italy).
The data of the survival curves were plotted as percentages of untreated controls. Each experiment consisted of at least three replicates.

\section{In vivo tumor growth}

Female Athymic Swiss NCr-nu/nu mice (4 weeks old) were obtained from Charles River (Calco, Italy). Mice were maintained under pathogen-free conditions, and provided food and water ad libitum.

Procedures involving animals and their care are conducted in conformity with the institutional guidelines that are in compliance with national (D.L. n.116,G.U., suppl. 40, 18 Febbraio 1992, Circolare No. 8, G.U., 14 Luglio 1994) and international laws and policies (EEC Council Directive 86/609, OJ L 358,1, Dec 12, 1987; Guide for the use of Laboratory Animals, United States National Research Council, 1996).

HCT-116/8a, HCT116/DN3 and HCT 116/DN14 tumor cells were transplanted in the left flank of the recipient mice $\left(5 \times 10^{6} \mathrm{cells} /\right.$ mouse $)$. At the beginning of the treatment with tetracycline, mice were divided randomly into test groups consisting of at least six mice each. Tetracycline was provided with drinking water $(2 \mathrm{mg} / \mathrm{ml})$ throughout the period of the experiment and was replaced every other day.

The length $(L)$ and the width $(W)$ of the tumor mass were measured by calliper twice weekly, and the tumor volume (TV) was calculated as: $\mathrm{TV}=\left(L \times W^{2}\right) / 2$. Tumor volumes of mice receiving or not tetracycline were compared using Fisher's test. Analyses were computed using the StatView statistical package by SAS Institute Inc. (Third edition). At the end of experiments animals were killed and tumor tissue excised. Fragments of the tumors were immediately frozen in liquid nitrogen for protein or RNA extraction and other pieces were fixed in formalin and in OCT solution for IHC analysis.

Activity of cDDP was evaluated in mice bearing the different tumors either in the presence or in the absence of tetracycline. CDDP was dissolved in sterile saline and administered intravenously at the dose of $4 \mathrm{mg} / \mathrm{kg}$ every 7 days for three times. The percentage of tumor growth inhibition $(T / C \%)$ was calculated for each time point as: $T / C \%=$ $100 \times$ (mean tumor weight of treated group)/(mean tumor weight of control group). The maximal inhibition for each treatment was achieved 7 days following the last treatment.

\section{Acknowledgements}

The generous contribution of the Italian Association for Cancer Research is gratefully acknowledged. This work has been partially supported by grant from Italian Health Ministry to MB and by Fondazione CARIPLO. MM is a fellow of the Monzino Foundation.

\section{References}

1. Kaghad M, Bonnet $H$, Yang A, Creancier L, Biscan JC, Valent A, Minty A, Chalon P, Lelias JM, Dumont X, Ferrara P, McKeon F and Caput D (1997) Monoallelically expressed gene related to $p 53$ at $1 \mathrm{p} 36$, a region frequently deleted in neuroblastoma and other human cancers. Cell 90: 809-819

2. Zhu J, Jiang J, Zhou W and Chen X (1998) The potential tumor suppressor p73 differentially regulates cellular p53 target genes. Cancer Res. 58: 5061-5065

3. Yang A, Walker N, Bronson R, Kaghad M, Oosterwegel M, Bonin J, Vagner C, Bonnet H, Dikkes P, Sharpe A, McKeon F and Caput D (2000) p73-deficient mice have neurological, pheromonal and inflammatory defects but lack spontaneous tumours. Nature 404: 99-103

4. Moll UM, Erster S and Zaika A (2001) p53, p63 and p73 - solos, alliances and feuds among family members. Biochim. Biophys. Acta 1552: 47-59 
5. Melino G, De Laurenzi V and Vousden KH (2002) p73: friend or foe in tumorigenesis. Nat. Rev. Cancer 2: 605-615

6. Levrero M, De Laurenzi V, Costanzo A, Gong J, Melino G and Wang JY (1999) Structure, function and regulation of p63 and p73. Cell Death Differ. 6 : 1146-1153

7. Ueda Y, Hijikata M, Takagi S, Chiba T and Shimotohno K (1999) New p73 variants with altered $\mathrm{C}$-terminal structures have varied transcriptional activities. Oncogene 18: 4993-4998

8. Ishimoto O, Kawahara C, Enjo K, Obinata M, Nukiwa T and Ikawa S (2002) Possible oncogenic potential of DeltaNp73: a newly identified isoform of human p73. Cancer Res. 62: 636-641

9. Zaika Al, Slade N, Erster SH, Sansome C, Joseph TW, Pearl M, Chalas E and Moll UM (2002) DeltaNp73, a dominant-negative inhibitor of wild-type p53 and TAp73, is up-regulated in human tumors. J. Exp. Med. 196: 765-780

10. Stiewe T, Zimmermann S, Frilling A, Esche $H$ and Putzer BM (2002) Transactivation-deficient DeltaTA-p73 acts as an oncogene. Cancer Res. 62: 3598-3602

11. Nakagawa T, Takahashi M, Ozaki T, Watanabe Ki K, Todo S, Mizuguchi H, Hayakawa $T$ and Nakagawara A (2002) Autoinhibitory regulation of p73 by delta Np73 to modulate cell survival and death through a p73-specific target element within the delta Np73 promoter. Mol. Cell. Biol. 22: 2575-2585

12. Vossio S, Palescandolo E, Pediconi N, Moretti F, Balsano C, Levrero M and Costanzo A (2002) DN-p73 is activated after DNA damage in a p53-dependent manner to regulate p53-induced cell cycle arrest. Oncogene 21: 3796-3803

13. Kartasheva NN, Contente A, Lenz-Stoppler C, Roth J and Dobbelstein M (2002) p53 induces the expression of its antagonist p73 delta N, establishing an autoregulatory feedback loop. Oncogene 21: 4715-4727

14. Stiewe T, Theseling CC and Putzer BM (2002) Transactivation-deficient Delta TA-p73 inhibits p53 by direct competition for DNA binding: implications for tumorigenesis. J. Biol. Chem. 277: 14177-14185

15. Fillippovich I, Sorokina N, Gatei M, Haupt Y, Hobson K, Moallem E, Spring K, Mould M, McGuckin MA, Lavin MF and Khanna KK (2001) Transactivationdeficient p73alpha (p73Deltaexon2) inhibits apoptosis and competes with p53. Oncogene 20: 514-522

16. Maisse C, Munarriz E, Barcaroli D, Melino G and De Laurenzi V (2004) DNA damage induces the rapid and selective degradation of the DeltaNp73 isoform, allowing apoptosis to occur. Cell Death Differ. 11: 685-687

17. Casciano I, Mazzocco K, Boni L, Pagnan G, Banelli B, Allemanni G, Ponzoni M, Tonini GP and Romani M (2002) Expression of DeltaNp73 is a molecular marker for adverse outcome in neuroblastoma patients. Cell Death Differ. 9: 246-251

18. Concin N, Becker K, Slade N, Erster S, Mueller-Holzner E, Ulmer $H$, Daxenbichler G, Zeimet A, Zeillinger R, Marth C and Moll UM (2004) Transdominant $\backslash\{$ Deltal\}TAp73 isoforms are frequently up-regulated in ovarian cancer. Evidence for their role as epigenetic p53 inhibitors in vivo. Cancer Res. 64: 2449-2460

19. Chi SG, Chang SG, Lee SJ, Lee CH, Kim JI and Park JH (1999) Elevated and biallelic expression of p73 is associated with progression of human bladder cancer. Cancer Res. 59: 2791-2793

20. Ejeskar K, Sjoberg RM, Kogner P and Martinsson T (1999) Variable expression and absence of mutations in p73 in primary neuroblastoma tumors argues against a role in neuroblastoma development [In Process Citation]. Int. J. Mol. Med. 3: 585-589

21. Loiseau H, Arsaut $\mathrm{J}$ and Demotes-Mainard $\mathrm{J}$ (1999) p73 gene transcripts in human brain tumors: overexpression and altered splicing in ependymomas. Neurosci. Lett. 263: 173-176

22. Mai M, Yokomizo A, Qian C, Yang P, Tindall DJ, Smith DI and Liu W (1998) Activation of p73 silent allele in lung cancer. Cancer Res. 58: 2347-2349

23. Yoshikawa $H$, Nagashima M, Khan MA, McMenamin MG, Hagiwara $K$ and Harris CC (1999) Mutational analysis of p73 and p53 in human cancer cell lines. Oncogene 18: 3415-3421

24. Bergamaschi D, Gasco M, Hiller L, Sullivan A, Syed N, Trigiante G, Yulug I, Merlano M, Numico G, Comino A, Attard M, Reelfs O, Gusterson B, Bell AK, Heath V, Tavassoli M, Farrell PJ, Smith P, Lu X and Crook T (2003) p53 polymorphism influences response in cancer chemotherapy via modulation of p73-dependent apoptosis. Cancer Cell 3: 387-402

25. Irwin MS (2004) Family feud in Chemosensitvity: p73 and mutant p53. Cell Cycle 3: 319-323

26. Grob TJ, Novak U, Maisse C, Barcaroli D, Luthi AU, Pirnia F, Hugli B, Graber HU, De Laurenzi V, Fey MF, Melino G and Tobler A (2001) Human delta Np73 regulates a dominant negative feedback loop for TAp73 and p53. Cell Death Differ. 8: 1213-1223

27. Casciano I, Ponzoni M, Cunsolo C, Tonini G and Romani M (1999) Different p73 splicing variants are expressed in distinct tumour areas of a multifocal neuroblastoma [in process citation]. Cell Death Differ. 6: 391-393

28. Petrenko O, Zaika A and Moll UM (2003) deltaNp73 facilitates cell immortalization and cooperates with oncogenic Ras in cellular transformation in vivo. Mol. Cell. Biol. 23: 5540-5555

29. Kartasheva NN, Lenz-Bauer C, Hartmann O, Schafer $H$, Eilers $M$ and Dobbelstein M (2003) DeltaNp73 can modulate the expression of various genes in a p53-independent fashion. Oncogene 22: 8246-8254

30. Liu G, Nozell S, Xiao H and Chen X (2004) DeltaNp73beta is active in transactivation and growth suppression. Mol. Cell. Biol. 24: 487-501

31. Gong JG, Costanzo A, Yang HQ, Melino G, Kaelin Jr WG, Levrero M and Wang JY (1999) The tyrosine kinase c-Abl regulates p73 in apoptotic response to cisplatin-induced DNA damage. Nature 399: 806-809

32. Bunz F, Hwang PM, Torrance C, Waldman T, Zhang Y, Dillehay L, Williams J, Lengauer C, Kinzler KW and Vogelstein B (1999) Disruption of p53 in human cancer cells alters the responses to therapeutic agents. J. Clin. Invest. 104: 263-269

33. Bunz F (2002) Human cell knockouts. Curr. Opin. Oncol. 14: 73-78

34. Vikhanskaya F, Colella G, Valenti M, Parodi S, D'Incalci M and Broggini M (1999) Cooperation between p53 and hMLH1 in a human colocarcinoma cell line in response to DNA damage. Clin. Cancer Res. 5: 937-941

35. Conforti G, Codegoni AM, Scanziani E, Dolfini E, Dasdia T, Calza M, Caniatti M and Broggini M (1995) Different vimentin expression in two clones derived from a human colocarcinoma cell line (LoVo) showing different sensitivity to doxorubicin. Br. J. Cancer 71: 505-511

36. Marabese M, Vikhanskaya F, Rainelli C, Sakai T and Broggini M (2003) DNA damage induces transcriptional activation of p73 by removing C-EBPalpha repression on E2F1. Nucleic Acids Res. 31: 6624-6632

\section{Supplementary Information accompanies the paper on Cell Death and Differentiation website (http://www.nature.com/cdd)}

